\title{
AVALIAÇÃO DO ESTADO NUTRICIONAL DE CRIANÇAS E ADOLESCENTES COM ASMA
}

\author{
Maria Ângela Góes Monteiro Antonio*, José Dirceu Ribeiro, Adyléia Aparecida Contrera Toro, \\ Aquiles Eugênico Piedrabuena, André Moreno Morcillo \\ Trabalho realizado no Hospital das Clínicas da Universidade Estadual de Campinas, SP.
}

RESUMO - OBjETIVo. Analisar o estado nutricional de crianças e adolescentes com asma em relação à gravidade, idade de início da doença, renda familiar per capita e escolaridade materna.

Métodos. Estudo transversal, no qual foram examinados 66 pacientes portadores de asma atópica do Ambulatório de Imunologia Alergia e Pneumologia do Hospital das Clínicas da Universidade Estadual de Campinas (HC/Unicamp), com idade entre 4 e 14 anos completos, e, posteriormente, foram comparados com 124 controles.

RESULTADOs. No sexo masculino, em relação à gravidade, idade de início da doença, renda familiar per capita e escolaridade materna, não se encontrou diferença estatisticamente significante na distribuição dos escores $z$ do peso/idade $(p=0,24)$, altura/idade $(p=0,6 I)$, índice de massa corporal $(p=0,2 I)$, perímetro braquial $(p=0,94)$ e prega cutânea tricipital $(p=0,49)$ entre casos e controles. Em relação ao sexo feminino, não se observou diferença estatisticamente significante para a distribuição dos escores $z$ do peso/ idade $(p=0,13)$, altura/idade $(p=0,46)$, índice de massa corporal $(p=0,13)$, perímetro braquial $(p=0,06)$, somente as medidas da prega cutânea tricipital dos casos $(-0,74 \pm 0,84)$ apresentaram valores estatisticamente diferentes $(p=0,002)$ dos controles
$(0,17 \pm I, 21)$. Nenhuma criança com asma apresentou obesidade (escore $\mathbf{z}$ do índice de massa corporal $\geq 2$ ). Entre os casos, não se observou diferença estatisticamente significante das medidas antropométricas, considerando-se a renda familiar per capita, escolaridade materna, gravidade da doença e idade de início, exceto para os meninos com asma moderada que apresentaram médias de peso inferior à dos demais $(p=0,02)$.

Conclusõos. Pode-se inferir que 0 aporte nutricional destes pacientes seja satisfatório, de tal forma que a composição corporal não foi afetada pela doença, pelo ambiente ou pela interação entre ambos.

Nossos resultados reforçam a opinião de que a avaliação antropométrica deve ser realizada periodicamente, pois esta rotina permite avaliar as conseqüências da enfermidade, 0 impacto das intervenções e das medidas terapêuticas empregadas e, conseqüentemente, a saúde geral do indivíduo.

UnITERMOS: Avaliação nutricional. Asma. Crescimento. Crianças. Adolescentes. Composição corporal.

\section{INTRODUÇÃO}

Aasma é uma doença inflamatória crônica de alta prevalência e tem sido considerada um problema de saúde pública'.

Há cerca de 60 anos, Cohen, Weller e Cohen ${ }^{2}$ observaram que algumas crianças alérgicas apresentavam parada do crescimento, que se manifestava inicialmente por perda de peso, e que, com a persistência dos sintomas, podiam ter sua estatura e maturidade óssea comprometidas. Oito anos mais tarde, esses mesmos autores confirmaram seus achados em um grupo de crianças em que a maioria era asmática, concluindo que a alergia ativa era a causa do retardo do crescimento e que este poderia ser revertido com o controle da mesma ${ }^{3}$.

* Correspondência: Av. Modesto Fernandes, $354-$ C. 3 Cep: 13085-472 - Campinas - SP
Desde então, a maioria dos estudos com pacientes asmáticos têm se preocupado em avaliar o efeito da doença e do uso de corticosteróides inalatórios sobre o crescimento linear com resultados variáveis ${ }^{4}$. Mais recentemente, a possibilidade da associação entre asma e obesidade tem sido pesquisada tanto em adultos como em crianças e adolescentes ${ }^{5-8}$.

Outros estudos procuraram determinar o impacto do nível socioeconômico sobre o crescimento dos pacientes com asma. No Brasil, Grumach et al ${ }^{9}$ observaram que a relação entre o nível socioeconômico e a gravidade da asma era altamente significante, Solé et al. ${ }^{10}$ ao estudarem crianças alérgicas da cidade de São Paulo, observaram que a doença alérgica isolada não foi a causa da baixa estatura, mas o nível socioeconômico desfavorável potencializou - retardo do crescimento e Sant'Anna et al. " observaram uma freqüência significa- tivamente maior de baixa estatura entre os atópicos, concluindo que, para aquela população, a atopia por si só era capaz de promover um déficit de crescimento.

$O$ objetivo deste trabalho foi analisar 0 estado nutricional de crianças e adolescentes com asma atópica em relação à gravidade, idade de início da doença, renda familiar per capita e escolaridade materna.

\section{Métodos}

Foram incluídos 66 crianças e adolescentes portadores de asma atópica, atendidos no Ambulatório de Imunologia, Alergia e Pneumologia Pediátrica do HC/UNICAMP e 124 controles de semelhante idade, escolaridade materna e renda familiar per capita, selecionados ao acaso de dois estudos com pré-escolares e escolares do município de Paulíinia,SP.

Foram excluídos os pacientes com malformação física e outras doenças associadas. 


\begin{tabular}{|c|c|c|c|c|c|}
\hline & & \multicolumn{2}{|c|}{ Masculino } & \multicolumn{2}{|c|}{ Feminino } \\
\hline & & Casos & Controles & Casos & Controles \\
\hline $\mathrm{n}$ & & 38 & 68 & 28 & 56 \\
\hline Idade* & & $8,79 \pm 2,47$ & $9,07 \pm 2,32$ & $8,37 \pm 1,92$ & $8,52 \pm 1,92$ \\
\hline \multirow[t]{4}{*}{ Rendaper capita } & $<0,5$ & I & 8 & 4 & 10 \\
\hline & $0,5-\mid 1,0$ & 14 & 22 & 9 & 15 \\
\hline & $1,0-2,0$ & 15 & 29 & 8 & 17 \\
\hline & $>2,0$ & 7 & 9 & 7 & II \\
\hline \multirow[t]{3}{*}{ Escolaridadematerna } & $\leq 4$ & 22 & 37 & 16 & 32 \\
\hline & $5 \overline{\mid}-8$ & 12 & 23 & 8 & 16 \\
\hline & $>8$ & 2 & 6 & 3 & 6 \\
\hline
\end{tabular}

n: número de casos

* média \pm desvio padrão

Tabela 2 - Distribuição da média e do desvio padrão (DP) dos escores z da altura/idade, do peso/idade, do índice de massa corporal (IMC), do perímetro braquial (PB) e da prega cutânea tricipital (PCT)

\begin{tabular}{|c|c|c|c|c|c|c|}
\hline \multirow[b]{2}{*}{ Escoresz $z^{*}$} & \multicolumn{3}{|c|}{ Sexo masculino } & \multicolumn{3}{|c|}{ Sexo feminino } \\
\hline & Casos & Controles & $\mathbf{p}$ & Casos & Controles & $\mathrm{p}$ \\
\hline & $n=38$ & $n=68$ & P & $n=28$ & $n=56$ & \\
\hline Altura & $0,13 \pm 1,03$ & $-0,13 \pm 1,19$ & 0,61 & $-0,29 \pm 1,08$ & $-0,003 \pm 0,88$ & 0,46 \\
\hline Peso & $0,28 \pm 1,33$ & $-0,14 \pm 1,13$ & 0,24 & $-0,39 \pm 0,61$ & $-0,06 \pm 1,05$ & 0,13 \\
\hline IMC & $-0,36 \pm 1,32$ & $-0,18 \pm 0,90$ & 0,21 & $-0,80 \pm 0,998$ & $-0,18 \pm 1,20$ & 0,13 \\
\hline PB & $-0,50 \pm 1,21$ & $-0,51 \pm 0,94$ & 0,94 & $-0,82 \pm 1,07$ & $-0,29 \pm 1,03$ & 0,06 \\
\hline PCT & $-0,32 \pm 0,89$ & $0,10 \pm 1,34$ & 0,49 & $-0,74 \pm 0,84$ & $0,|7 \pm|, 2 \mid$ & 0,002 \\
\hline
\end{tabular}

n: número de pacientes; p:probabilidade do teste de Kolmorov-Smirnov

*média \pm desvio padrão

O estudo foi aprovado pelo Comitê de Ética em Pesquisa do HC-UNICAMP e todos os pacientes tiveram o consentimento de seus pais ou responsáveis.

Os pacientes foram submetidos a um exame antropométrico que incluiu as medidas da altura, do peso, do perímetro braquial (PB) e da prega cutânea tricipital, (PCT). As medidas foram realizadas empregando-se as técnicas propostas por Cameron ${ }^{12}$.

Aárea muscular braquial (AMB) foi calculada a partir do perímetro braquial e da prega cutânea tricipital, utilizando-se as fórmulas de Frisancho ${ }^{13}$. Oíndice de massa corporal (IMC) foi estimado a partir do peso e da altura.

No cálculo dos escores $z$ do peso/idade e altura/idade, utilizou-se a curva do National Center for Health Statistics 14,15 ; para o IMC, os dados de Must ${ }^{16}$; para o PB, PCT, AMB e AGB os dados de Frisancho ${ }^{13}$.

A renda familiar per capita foi calculada a partir da divisão da renda familiar mensal pelo número de pessoas que dela usufruíame transformada em salários-mínimos (SM), formando os seguintes agrupamentos: $\leq 0,5 \mathrm{SM} ; 0,5-1$ I,00 SM; I,00 -| 2,00 SM e > 2,00 SM.
A escolaridade da mãe foi assim agrupada: $\leq 4$ anos - mães que tinham completado atéa $4^{\mathrm{a}}$ série do $I^{\circ}$ grau; $5|-| 8$ anos - mães que tinham completado da $5^{\mathrm{a}}$ a $8^{\mathrm{a}}$ série do $\mathrm{I}^{\circ} \mathrm{grau}$ ou supletivo do $I^{\circ}$ grau; $>8$ anos - mães que tinham completado da $I^{\mathrm{a}}$ a $3^{\mathrm{a}}$ série do $2^{\circ} \mathrm{grau}$ ou supletivo do $2^{\circ}$ grau no mínimo.

Para definição da gravidade da asma no início do estudo, atendeu-se o estabelecido no $3^{\circ}$ Consenso Internacional ${ }^{17}$.

Aidade de início das crises de asma foiassim agrupada: menos que I ano: pacientes que tiveram o primeiro episódio de asma durante 0 primeiro ano de vida; I a 3 anos: pacientes que tiveram o primeiro episódio de asma entre 0 segundo e o terceiro ano de vida; mais de 3 anos: pacientes que tiveram o primeiro episódio de asma a partir do quarto ano de vida.

\section{Análise estatística}

$\mathrm{Na}$ comparação dos escores $z$ das variáveis antropométricas entre os casos e controles utilizou-se o teste de Kolmorov-Smirnnov. Para a comparação da distribuição dos escores z em relação à gravidade, idade de início da doença, renda familiar per capita e escolarida- de materna, empregou-se o teste de KruskalWallis ${ }^{18,19}$. Em todos os casos adotou-se o nível de significância de 0,05.

\section{Resultados}

A caracterização dos casos e controles com relação à idade, sexo, escolaridade da mãe e renda familiar per capita é apresentada na Tabela I. Quanto à gravidade da asma, 17 (25,7\%) tinham asma leve; 18 (27,3\%), moderada e 3I, (47\%) grave.

No sexo masculino, em relaçãoà gravidade, idadede início da doença, rendafamiliarper capita e escolaridade materna, não se encontrou diferença estatisticamente significantena distribuição dos escores $z$ do peso/idade $(p=0,24)$, altura/ idade $(p=0,61), I M C(p=0,21), P B(p=0,94) e$ $\operatorname{PCT}(p=0,49)$ entre casos e controles. No sexo feminino tambémnãose observou diferençaestatisticamente significante paraa distribuição dos escores $z$ do peso $(p=0,13)$, altura $(p=0,46)$, $\operatorname{IMC}(p=0,13), P B(p=0,06)$. Quanto à $P C T$, os casos $(-0,74 \pm 0,84)$ apresentaram valores estatisticamente diferentes $(p=0,002)$ dos controles $(0,17 \pm I, 2 I)($ Tabela 2$)$. 
Tabela 3 - Valores da média e do desvio-padrão (DP) dos escores z da altura/idade, do peso/idade, do índice de massa corporal (IMC), da prega cutânea tricipital (PCT) e da área muscular braquial (AMB) das meninas, em relação à renda familiar per capita (em salário-mínimo), escolaridade materna (em anos), gravidade e idade de início da doença

\begin{tabular}{|c|c|c|c|c|c|c|c|c|c|c|c|c|}
\hline & & & Altura & & Peso & & IMC & & РCT & & AMB & \\
\hline & & $\mathrm{n}$ & média $\pm \mathrm{D}$ & $\mathbf{P}$ & média $\pm \mathrm{DP}$ & $p$ & média $\pm \mathrm{DP}$ & $p$ & média $\pm \mathrm{DP}$ & $p$ & média $\pm \mathrm{DP}$ & $p$ \\
\hline Renda & $<0,5$ & $\overline{4}$ & $-0,12 \pm 0,87$ & & $-0,58 \pm 0,29$ & & $-1,32 \pm 0,49$ & & $-1,03 \pm 0,67$ & & $-1,25 \pm 1,30$ & \\
\hline \multirow{3}{*}{ percapita } & $0,5-\mid 1,00$ & 9 & $-0,04 \pm 1,47$ & & $-0,16 \pm 0,61$ & & $-0,62 \pm 0,96$ & & $-0,72 \pm 0,70$ & & $-0,36 \pm 0,91$ & \\
\hline & $1,0-\mid 2,00$ & 8 & $-0,34 \pm 0,90$ & 0,49 & $-0,30 \pm 0,73$ & 0,17 & $-0,51 \pm 1,08$ & 0,37 & $-0,35 \pm 0,98$ & 0,45 & $-0,39 \pm 0,77$ & 0,50 \\
\hline & $>2,00$ & 7 & $-0,65 \pm 0,90$ & & $-0,67 \pm 0,54$ & & $-1,06 \pm 1,11$ & & $-1,05 \pm 0,91$ & & $-0,71 \pm 1,12$ & \\
\hline \multirow[t]{3}{*}{ Escolaridade } & $\leq 4$ & 16 & $-0,20 \pm 1,16$ & & $-0,39 \pm 0,56$ & & $-0,95 \pm 0,94$ & & $-0,90 \pm 0,83$ & & $-0,70 \pm 0,96$ & \\
\hline & $5|-| 8$ & 8 & $-0,30 \pm 1,13$ & & $-0,37 \pm 0,80$ & & $-|, 0| \pm 0,98$ & & $-0,73 \pm 0,97$ & 0,17 & $-0,79 \pm 0,84$ & \\
\hline & $>8$ & 3 & $-0,85 \pm 0,85$ & 0,57 & $-0,41 \pm 0,66$ & 0,99 & $0,48 \pm 0,43$ & 0,09 & $0,02 \pm 0,14$ & & $0,31 \pm 1,35$ & 0,33 \\
\hline \multirow[t]{2}{*}{ Gravidade } & leve & 5 & $-0,15 \pm 0,47$ & & $0,21 \pm 0,14$ & & $-0,23 \pm 0,44$ & & $-0,25 \pm 0,74$ & & $-0,32 \pm 1,00$ & \\
\hline & $\begin{array}{l}\text { moderada } \\
\text { grave }\end{array}$ & $\begin{array}{l}12 \\
11\end{array}$ & $\begin{array}{l}-0,18 \pm 1,24 \\
-0,47 \pm 1,15\end{array}$ & 0,74 & $\begin{array}{l}-0,37 \pm 0,69 \\
-0,48 \pm 0,66\end{array}$ & 0,46 & $\begin{array}{l}-0,73 \pm 1,04 \\
-1,13 \pm 1,03\end{array}$ & 0,19 & $\begin{array}{l}-0,69 \pm 0,74 \\
-1,02 \pm 0,95\end{array}$ & 0,11 & $\begin{array}{l}-0,39 \pm 1,04 \\
-0,91 \pm 0,90\end{array}$ & 0,42 \\
\hline \multirow[t]{3}{*}{ Ińício } & $<1$ & 10 & $-0,50 \pm 1,36$ & & $-0,33 \pm 0,78$ & & $-0,56 \pm 1,08$ & & $-0,69 \pm 0,93$ & & $-0,20 \pm 0,63$ & \\
\hline & $|-| 3$ & 9 & $-0,008 \pm 0,85$ & 0,63 & $-0,41 \pm 0,53$ & 0,97 & $-1,02 \pm 0,74$ & 0,67 & $-0,72 \pm 0,88$ & 0,94 & $-0,84 \pm 0,74$ & 0,17 \\
\hline & $3-16$ & 9 & $-0,35 \pm 0,99$ & & $-0,43 \pm 0,53$ & & $-0,84 \pm 1,12$ & & $-0,82 \pm 0,80$ & & $-0,75 \pm|, 4|$ & \\
\hline
\end{tabular}

n: númerodecasos; p: probabilidade do testede Kruskall-Wallis

Tabela 4 - Valores da média e do desvio padrão (DP) dos escores z da altura/idade, do peso/idade, do índice de massa corporal (IMC), da prega cutânea tricipital (PCT) e da área muscular braquial (AMB) dos meninos, em relação à renda familiar per capita (em salário-mínimo), escolaridade materna (em anos), gravidade e idade de início da doença

\begin{tabular}{|c|c|c|c|c|c|c|c|c|c|c|c|c|}
\hline & \multicolumn{3}{|c|}{ Altura } & \multicolumn{2}{|l|}{ Peso } & \multicolumn{2}{|l|}{ IMC } & \multicolumn{2}{|l|}{ PCT } & \multicolumn{2}{|l|}{ AMB } \\
\hline & & $\mathrm{n}$ & média $\pm \mathrm{D}$ & $\mathbf{P}$ & média $\pm \mathrm{DP}$ & $p$ & média $\pm \mathrm{DP}$ & $p$ & média $\pm \mathrm{DP}$ & $p$ & média $\pm D P$ & $p$ \\
\hline Renda & $<0,5$ & I & I,II & & $\begin{array}{c}0,41 \\
0,1+17\end{array}$ & & $\begin{array}{c}-0,48 \\
-0839+|4|\end{array}$ & & $\begin{array}{c}-0,54 \\
-0,56+097\end{array}$ & & & \\
\hline \multirow[t]{3}{*}{ percapita } & $0,5-||, 00$ & 14 & $-0,26 \pm 1,34$ & & $-0,21 \pm 1,37$ & & $-0,839 \pm 1,41$ & & $-0,56 \pm 0,97$ & & $-0,90 \pm 1,13$ & 0,29 \\
\hline & $1,0-\mid 2,00$ & 15 & $0,30 \pm 0,84$ & 0,41 & $0,32 \pm 1,03$ & 0,26 & $-0,29 \pm 1,06$ & 0,35 & $-0,31 \pm 0,64$ & 0,84 & $-0,29 \pm 1,28$ & \\
\hline & $>2,00$ & 7 & $0,33 \pm 0,56$ & & $0,78 \pm 1,48$ & & $0,08 \pm 1,48$ & & $-1,17 \pm 0,92$ & & $-0,15 \pm 0,86$ & \\
\hline \multirow[t]{3}{*}{ Escolaridade } & $\leq 4$ & 22 & $0,36 \pm 0,87$ & & $0,15 \pm 1,20$ & & $-0,52 \pm|, 2|$ & & $-0,40 \pm 0,80$ & & $-0,49 \pm 1,16$ & 0,16 \\
\hline & $5|-| 8$ & 12 & $-0,04 \pm 1,05$ & 0,37 & $0,75 \pm 1,57$ & 0,30 & $0,09 \pm 1,54$ & 0,28 & $-0, I I \pm I, 14$ & 0,61 & $0,03 \pm 1,12$ & \\
\hline & $>8$ & 2 & $-1,22 \pm 2,47$ & & $-0,85 \pm 1,56$ & & $-|, 32 \pm|, 3 \mid$ & & $-0,86 \pm 0,48$ & & $-1,86 \pm 1,19$ & \\
\hline \multirow[t]{3}{*}{ Gravidade } & leve & 12 & $0,52 \pm 0,70$ & & $0,81 \pm 1,13$ & & $0, I I \pm I, I I$ & & $-0,05 \pm 0,83$ & & $0,02 \pm 0,80$ & \\
\hline & moderada & 6 & $0,01 \pm 0,83$ & 0,36 & $-0,33 \pm 1,25$ & 0,02 & $-0,47 \pm|, 0|$ & 0,22 & $-0,60 \pm 1,07$ & 0,37 & $-0,2| \pm|, 0 \mid$ & 0,20 \\
\hline & grave & 20 & $-0,07 \pm 1,21$ & & $-0,15 \pm|, 4|$ & & $-0,62 \pm 1,49$ & & $-0,40 \pm 0,88$ & & $-0,72 \pm 1,34$ & \\
\hline \multirow[t]{3}{*}{ Início } & $<1$ & 21 & $0,22 \pm 0,80$ & & $0,30 \pm 1,115$ & & $-0,29 \pm 1,23$ & & $-0,25 \pm 0,84$ & & $-0,29 \pm 1,20$ & \\
\hline & $1-\mid 3$ & 10 & $0,09 \pm 1,36$ & 0,61 & $0,17 \pm 1,64$ & 0,76 & $-0,56 \pm 1,63$ & 0,52 & $-0,71 \pm 1,05$ & 0,17 & $-0,46 \pm 1,27$ & 0,58 \\
\hline & $3-16$ & 6 & $-0,29 \pm 1,24$ & & $0,15 \pm 0,63$ & & $-0,67 \pm 0,96$ & & $-0,17 \pm 0,48$ & & $-0,94 \pm 0,85$ & \\
\hline
\end{tabular}

n: número decasos; : probabilidadedo testede Kruskall-Wallis

Nenhuma criança com asma apresentou obesidade (escore $z$ de $P C T \geq 2$ ), enquanto $8,9 \%$ das crianças do grupo controle tinham escore $z$ de $P C T \geq 2$.

$\mathrm{Na}$ análise das medidas antropométricas dos casos, considerando a renda familiar per capita, escolaridade materna, gravidade da doença e idade de início, não se observou diferença estatisticamente significante, exceto para os meninos com asma moderada que apresentaram médias de peso inferior à dos demais $(p=0,02)$ (Tabelas 3 e 4).

\section{Discussão}

Este estudo foi realizado em um hospital universitário que é referência para a região. $O$ Ambulatório de Imunologia, Alergia e Pneumologia Pediátrica recebe pacientes, encaminhados principalmente peloSUS ${ }^{20}$, havendo 
um predomínio de doenças graves, evidenciado na população estudada, da qual $74,2 \%$ eram crianças e jovens portadores de asma moderada e grave.

É conhecido que a asma moderada e grave, independentemente do tratamento, repercute sobre o crescimento, atrasando 0 estirão pubertário e ocorrendo, posteriormente, uma recuperação em relação à altura final $^{21,22,23}$. A grande variabilidade do quadro clínico e da sua terapêutica dificultam discernir entre os fatores responsáveis pela desaceleração do crescimento ${ }^{24,25,26}$.

Nossos resultados mostraram um grupo de crianças e adolescentes normais do ponto de vista nutricional quando comparados aos controles e a população de referência, nos levando a inferir que a doença e as condições de vida destes pacientes provavelmente não interferiram sobre o seu estado nutricional. A diferença observada na PCT das meninas poderia estar relacionada com um comprometimento nutricional, no entanto tal achado merece ser melhor observado.

Nogrupo comasma, agravidade, a idade de início, a escolaridade materna e a renda familiar per capita não foram associadas com déficit de altura e peso. Os resultados da literatura são variáveis, possivelmente pela interrelação entre a gravidade e a idade de início da doença com os fatores individuais e ambientais que são responsáveis pelo crescimento ${ }^{24,27-31}$.

As deficiências de crescimento, principalmente em países em desenvolvimento, estão, na maioria das vezes, relacionadas com deficiências nutricionais, tendo ligação direta com o nível socioeconômico da população. Neste estudo não foi possível associar uma pior nutrição com o nível socioeconômico.

Não encontramos casos de obesidade, achado que difere da literatura que vem relatando um aumento de obesos nos estudos com pacientes com asma ${ }^{6,8}$.

Pode-se inferir que, o aporte nutricional destes pacientes está sendo satisfatório, de tal forma que a composição corporal destes pacientes não foi afetada pela doença, pelo ambiente ou pela interação entre ambos.

Nossos resultados reforçam a opinião de que a avaliação antropométrica deve ser realizada periodicamente, pois este procedimento permite avaliar as conseqüências da enfermidade, o impacto das intervenções e das medidas terapêuticas empregadas e, conseqüentemente, a saúde geral do indivíduo.

\section{Agradecimentos}

Os autores agradecem a todos os colaboradores, especialmente ao prof. dr. Edgard Ferro Collares.

\section{SUMMARY}

EVALUATION OF THE NUTRITIONAL STATE OF THE CHILDREN AND ADOLESCENTS WITH ASTHMA

OBJECTIVE. The aim of this study was to analyze the nutritional state of children and adolescents with asthma, and to relate this to patient age at the onset of disease, severity of the disease, family per capita income, and the level of maternal schooling.

METHODS. A cross sectional study involved 66 patients from he Immunology, Allergy and Pulmonology outpatient clinic of the Hospital das Clinicas of the State University of Campinas ( $\mathrm{HCl}$ Unicamp). All of the patients suffered from atopic asthma and ranged in age from 4 to 14 years old. The patients were compared to 124 controls.

RESULTS. There were no differences among male patients and the controls in the distribution of the z-scores for weight/age $(p=0.24)$, height/age $(p=0.6 I)$, body mass index $(p=0.21)$, upper arm circumference $(p=0.94)$ and triceps skinfold $(p=0.49)$ in relation to disease severity, age at the first occurrence of disease, family per capita income, and the level of maternal schooling. Similarly, there were no significant differences in the $z$-scores for weight/age $(p=0.13)$, height/age $(p=0.46)$, body mass index $(p=0.13)$, upper arm circumference $(p=0.06)$ among female patients and their controls. However, there was a difference $(p=0.002)$ between the triceps skinfold of female patients $(-0.74 \pm 0.84)$ and the controls $(0.17 \pm 1.21)$. No child with asthma was obese (z-scores for body mass inde ${ }^{3} 2$ ). There were no significant differences in of the anthropometrical measurements among patients in relation to family per capita income, the level of maternal schooling, severity of the disease, and age at first occurrence the disease, but the mean weight of boys suffering from moderate asthma was lower than that of the other patients $(p=0.02)$.

ConcLusions. The nutritional status of the patients studied was satisfactory so that their body composition was unaffected by the disease, on environment, or by any interaction between these two factors. Our results reinforce the view that an anthropometrical evaluation should be done periodically to assess the consequences of the disease and the impact of any interventions and therapeutic measures used. This will provide a better evaluation of the patient's overall health. [Rev Assoc Med Bras 2003; 49(4): 367-7I]

KeY wORDS: Asthma. Growth. Children. Adolescent. Body Composition.

\section{REFERÊNCIAS}

I. CDC (Centers for Disease Control and Prevention). Surveillance for asthma-United States, I960-1995. MMWR 1998, 47(SS-I).

2. Cohen MB, Weller RR, Cohen S. Antropometry in children. Progress in allergic children as shown by increments in height, weight and maturity. Am J Dis Child 1940; 60:1058-66.

3. Cohen MB, Abram LE. Growth patterns of allergic children. JAllergy 1948; 19: 165-171.

4. Price J, Hindmarsh P, Hughes S, Efthimiou J. Evaluating the effects of asthma therapy on childhood growth: what can be learnt from the published Iliterature?. Eur Respir J 2002; 19:1 179-93.

5. Luder E, Melnick TA, DiMaio M. Association of being overweight with greater asthma symptoms in inner-city black and hispanic children. J Pediatr 1998; I 32:699-703.

6. Gennuso J, Epstein LH, Paluch RA, CernyF. The relationship between asthma and obesity in urban minority children and adolescents. Arch Pediatr Adolesc Med 1998; I 52: I I 97-200.

7. Chinn S, Rona RJ. Canthe increase in body mass index explain the rising trend in asthma in children? Thorax 200 I; 56:845-50.

8. Figueroa-MunozJl, ChinnS, Rona RJ. Association between obesity and asthma in 4-I I year old children in the UK. Thorax 200 I; 56: I 33-7.

9. Grumach AS, Carneiro-Sampaio MMS, Lima JL, Regis MJC, Marcondes E. Curva de crecimiento en niños asmáticos. Allergol Immunopathol (Madr) 1985; I 3:22 I-8.

10. Solé D, Scalabrin DMF, Sano F, Mallozi MC, Naspitz CK, Spínola-Castro AM, et al. Doença alérgica e sua repercussão sobre o crescimento. J Pediat (Rio de J) 1991; 67:92-100.

II. Sant'anna CA, Solé D, Naspitz CK. Short stature in children with respiratory allergy. Pediatr Allergy Immunol 1 996; 7: 187-92.

12. Cameron N. The methods of auxological anthropometry. In: Falkner F, Tanner JB ed. Human growth. New York: Plenum Press; 1978. p.35-90.

13. Frisancho AR. New norms of upper limb fat and muscle areas for assessment of nutritional status. Am J Clin Nutr 198I; 34:2540-5.

14. NCHS - National Center for Health Statistics. NCHS growth curves for children birth - 18 years. United States: vital and health statistics. Washington; 1977. (Series II, $\mathrm{n}^{\circ}$ 165). 
15. Organizacion Mundial de la Salud. Medición del cambio del estado nutricional. Genebra: OMS; 1983.

16. Must A, Dallal GE, Dietz WH. Reference data for obesity: $85^{\text {th }}$ and $95^{\text {th }}$ percentiles of body mass index $\left(\mathrm{w} / \mathrm{t}^{2}\right)$ and triceps skinfold thickness. Am J Clin Nutr 199 I; 54:773.

17. Warner JO, Naspitz CK. Third International Pediatric Consensus Statement on the management of childhood asthma. Pediatr Pulmonol 1998; 25: I-I 7 .

18. LevinJ. Estatística aplicada a ciências humanas. $2^{\mathrm{a}}$ ed. São Paulo: Ed. Harper Row do Brasil; 1985.

19. Siegel S. Estatística não-paramétrica - para ciências do comportamento. Recife: McGrawHill do Brasil; 1975.

20. Franco SC. Estudo da clientela, do sistema de referência e de indicadores de qualidade do Ambulatório de Pediatria do Hospital das Clínicas da Universidade Estadual de Campinas [dissertação]. Campinas: Faculdade de Ciências Médicas, Universidade Estadual de Campinas; 1995.
2I. Hauspie R, Susanne C, Alexander F. A mixed longitudinal study of the growth in height and weight in asthmatic children. Hum Biol 1976; 48:27I-83.

22. Hauspie R, Susanne C, Alexander F. Maturational delay and temporal growth retardation in asthmatic boys. J Allergy Clin Immunol I 977; 59:200-6.

23. Preece MA, Law CM, Davies PSW. The growth of children with chronic paediatric disease. Clin Endocrinol Metabol 1986; I 5:453-77.

24. Dawson B, Horobin G, Illsley R, Mitchell R. A survey of childood asthma in Aberdden. Lancet 1969; I:827.

25. Solé D, Spínola-Castro AM, Naspitz C K. Growth in allergic children. J Asthma 1989; 26:217-2I.

26. Solé D, Naspitz C K, Spínola-Castro AM, Denardin O. Atopia e retardo de crescimento: revisão. Rev Bras Alergia Imunopatol 1990; 13:19-23.

27. Falliers CJ, lan LS, Szentivanyi J, Jorgensen J,
Bukantz SC. Childhood asthma and steroid therapy as influences on growth. Am J Dis Child 1963; 105: I 27-37.

28. McNicol KN, Willians HB. Spectrum of asthma in children I. Clinical and physiological components. BMJ 1973; 4:7-II.

29. Gillam GL, McNicol, KN, Williams HE. Chest deformity, residual airways obstruction and hyperinflation, and growth in children with asthma II: significance of chronic chest deformity. Arch Dis Child 1970; 45:789-99.

30. Murray AB, Fraser BM, Hardwick DF, Pirie GE. Chronic asthma and growth failure in children. Lancet I976; 2:197-8.

3 I. Wittig HJ, McLaughlin ET, Belloit JD. Growth retardation with chronic asthma in the absence of prolonged steroid therapy. Allergol Immunopathol (Madr) 1978, 6:203-8.

Artigo recebido: 15/08/2002 Aceito para publicação: 03/04/2003

\section{Ante ஜ̈rasileira}

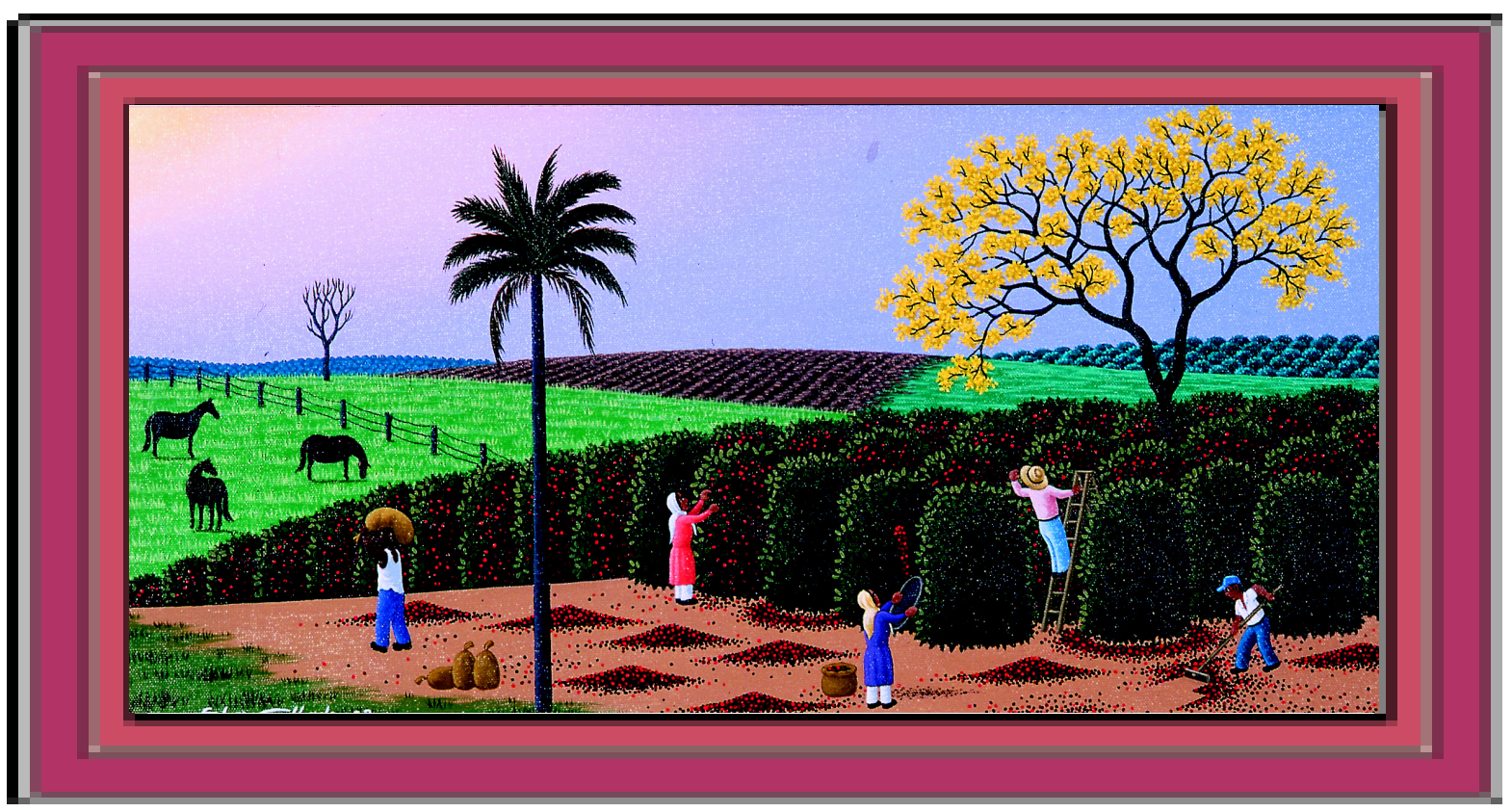

Edgar Calhado - "Cafezal, Sol e Ipê" - Galeria Jacques Ardies - Tel.: (I I) 3884-29I6 